\title{
PARASITIC AMEBAS IN THE INTESTINE OF MAN, WITII \\ A STUDY OF THE PROTOZOA FOUND IN THE \\ INTESTINES OF HEALTHY MEN IN THE SOUTHERN UNITED STATES
}

PRELIMINARY NOTE

EUGENE R. WHITMORE, M.D.

FORT BARRANCAS, FLA.

During the past few years, the parasitic protozoa have received a great deal of attention, and now the list of diseases known to be caused by protozoa has become a long one. Through all of this, the question has frequently been raised whether the protozoa found in certain diseases were really the cause of the disease or whether they were harmless parasites, their finding being purely coincidental.

And nowhere has the question of pathogenicity or non-pathogenicity been raised more frequently than in connection with the parasitic protozoa in the intestine of man. Thus, very recently, one worker has studied the stools and the intestinal lesions in a series of cases of amebic dysentery, and on the basis of this study he maintains that amebas do not cause dysentery, but that their presence, even in the ulcers and in the tissues, is purely accidental. On the other hand, we have the men who consider that all amebas are, or may become, pathogenic when taken into the intestinal tract of man.

The question of the pathogenicity or non-pathogenicity of the flagellata found in the intestine of man is not yet settled to the satisfaction of everybody. Early workers had shown conclusively that both pathogenic and non-pathogenic amebas were parasitic in the intestine of man, but it remained for Schaudinn to show how it was possible to distinguish between the two. He described a non-pathogenic ameba-Entamcba coli-commonly parasitic in the intestine of man, and a pathogenic ameba - Entamcba histolytica - found as the cause of dysentery in man. His work was accepted, and $E$. coli was shown to be a very common parasite in the intestine of healthy men in all parts of the world. E. histolytica was generally the ameba found in dysentery, though there have been occasional reports of $E$. coli in amebic dysentery and in liver abscesses.

But a later contribution to the study of the amebas parasitic in the intestine of man has not received the attention that it merits in this country. Schaudinn was aware that there was a third ameba parasitic 
in the intestine of man, but his untimely death left it for his pupil, Viereck, to work out this ameba and to give it the name Entamoba tetragena on account of the cyst with four nuclei.

Almost at the same time, Hartmann described a dysentery ameba found in the stools of cases of dysentery from Africa, but as he had only vegetative forms of the ameba to work with, he did not see the cysts with four nuclei, and so called his ameba Entamoba africana. Soon after this, Hartmann had a chance to work over some of Viereck's preparations, and he found that $E$. africana was E. tetragena, so africana fell as a species name.

$E$. tetragena resembles $E$. histolytica in the living state in that when at rest there is a distinct separation into ectoplasm and entoplasm, while it resembles $E$. coli in that the nucleus has a distinct nuclear membrane and is plainly visible in the living state.

In the vegetative forms of $E$. tetragena the nucleus, when properly stained, shows a central caryosome of variable size in which one can see a centriole. There is a bright zone around the caryosome, and this zone is marked off from the outer nuclear zone by a granular zone which is the original boundary of the caryosome. One often sees two such zones placed one inside the other. This distinct appearance of the cyclic change in the caryosome is very characteristic for $E$. tetragena. Indeed, Hartmann had only vegetative amebas to work with when he described his new ameba, and the most important point was this appearance of the nucleus.

But tetragena is distinctly marked off from both coli and histolytica in its cyst formation - the cyst with the dense sausage-shaped chromidia and four nuclei.

There is one great source of error: Under the conditions that cause tetragena to encyst, there are always a great many degenerating amebas in which the nucleus is in all stages of disintegration, and great care is necessary in order not to misinterpret such findings. Again, at this time the chromatin is passing off from the nucleus into the plasma to form the chromidia, and such an appearance may easily be mistaken for the nuclear change which takes place in histolytica preparatory to budding. Tast winter, while working in Professor Hartmann's laboratory, I had an opportunity to study the original preparations from a case of amebic dysentery that has been figured and described in some German text-books as a case of $E$. histolytica infection. Study of some sections freshly stained with iron hematoxylin showed that this was a case of E. tetragena infection.

Strangely enough, the common parasitic ameba of the intestine of the frog (E. ranarum) resembles $E$. tetragena and forms a cyst with four 
nuclei, while the common parasitic ameba of the intestine of the mouse (E. muris) resembles $E$. coli very closely and forms a cyst with eight nuclei.

Prowazek has recently described a new ameba from Samoa. It resembles coli very closely, but he finds sufficient differences to make it a new species.

Since the description of $E$. tetragena, it has been found to be the common dysentery ameba in all parts of the world in which it has been studied, while $E$. histolytica is found to be rare as a cause of dysentery. Hartmann says of E. tetragena that "it is far the more frequent of the two dysentery amebas (South America, Africa, India, etc.) ;" and again, he says of $E$. histolytica that "as a rule it is met with only in cases of dysentery from East Asia."

$E$. tetragena has been shown to be a cause of dysentery in China, French Indo-China, the Philippine Islands and Japan (Entamaba nipponica is undoubtedly E. tetragena); and in the large amount of material which I took to Berlin with me from Manila and Saigon, there was not a case of $E$. histolytica infection, all being tetragena or coli. Whether it happened that I did not get the cases of histolytica infection in collecting my material in Manila, or whether histolytica is rarer as a cause of dysentery in Manila than it was a few years ago, I am unable to say.

The great frequency of certain parasitic protozoa in the intestine of healthy men in the tropics is well known, but as is usual, it has not been so well worked out in the southern part of our own country.

King studied the prevalence of amebic dysentery in the southern United States, and from the information gathered by him, he decides that it is a rare disease here.

E. histolytica is generally the parasite found in the cases of amebic dysentery collected by King, though in a few cases $E$. coli is reported as the parasite found.

$E$. tetragena resembles $E$. coli very closely, and undoubtedly the cases of dysentery and liver abscess in which $E$. coli is reported as the cause are cases in which $E$. tetragena has been mistaken for $E$. coli or in which $E$. coli was present in addition to the dysentery ameba. Again, since $E$. coli is a very common parasite in the intestine of healthy persons in tropical and subtropical regions, it is possible that some of these cases were of bacillary or other origin, and the finding of $E$. coli was purely coincidental.

An interesting case of this nature has occurred in my series here. A young woman had a typical case of typhoid fever with positive blood culture and positive Widal. She had mild diarrhea throughout her attack of typhoid. Her stool was examined during this time and found 
to contain numerous vegetative forms and a few incomplete cysts of E. coli, and literally to swarm with Trichomonas intestinalis. Since her recovery her bowel movements are normal but cysts of $E$. coli and of $T$. intestinalis are still present in abundance. Another interesting point in this case was that many of the vegetative amebas contained, besides the nucleus, from one to several spherical bodies that might have been mistaken for red blood-cells, except that they were colorless. Stained with iron hematoxylin, these bodies proved to be cysts of $T$. intestinalis which had been ingested by the amebas.

King further found that amebas were not very commonly seen in routine examination of stools; thus in the recent examination of $40 \mathrm{~F}$ stools of the inmates of the Florida Insane Asylum located at Chattahoochee, amebas were not once demonstrable.

In order to add a little to the subject, I have undertaken the routine examination of the stools of the persons living in a settlement near here. These people are mostly negroes, and all are living "close to nature."

In the examination, several fresh smears from each stool are examined, and when any protozoa are found, smears are fixed with sublimatealcohol and stained with iron hematoxylin in the usual way. The final identification of the protozoa is made from these stained preparations.

In the stools of the first 100 persons examined, I found $E$. coli fiftyseven times and $T$. intestinalis forty-two times, the two parasites being together twenty-six times. Strangely, Lamblia was not found at all, and none of the rarer parasitic protozoa-Prowazekia, Balantidium, or Nyctotherus - was found.

Different observers have found amebas with or without intestinal ulcers in cases of pellagra, and the question of a relationship between pellagra and parasitic amebas has been raised.

I have recently had opportunity to study the stools of two cases of pellagra. Amebas were present in the stools of both cases and in both cases they proved to be $E$. coli. Trichomonas intestinalis was also present in the stools of one case. Both of these patients had severe diarrhea one of them having as many as thirty bowel movements in twenty-four hours. The latter patient died but a necropsy was not allowed.

My finding in these two cases of pellagra shows that it is not sufficient to find amebas in cases of pellagra; it is necessary to determine what species of ameba is present, by the study of properly fixed and stained specimens, before anything definite can be learned regarding the relationship between pellagra and parasitic amebas.

My finding of so high a percentage of infections with $E$. coli and $T$. intestinalis in healthy men seems to be rather out of accord with the findings of other workers in the southern United States, but from what we know of the finding for tropical and subtropical countries in general, 
this high percentage of infections is rather to be expected. Whether a larger number of examinations will reduce this percentage, or whether I have merely found a settlement where over half of the people are infected with $E$. coli, can only be determined by further work. The work will be continued and at a later date $I$ shall hope to give the percentage of infections for a larger series of cases.

From the known wide distribution of $E$. tetragena as a cause of dysentery, it is very probable that it is the cause of at least a part of the amebic dysentery in the United States, aside from the cases of this infection which are brought from the Philippine Islands and from South America. This is rendered all the more probable by the cases collected by King, in which $E$. coli is reported as the cause of amebic dysentery and liver abscess, as these cases must have been cases of $E$. tetragena infection.

\section{SUMMARY}

As this is a preliminary note, it is not safe to draw any conclusions until more work is done; but the work may be summed up in a few paragraphs, the points in which seem to be justified.

1. Entamcba coli and Trichomonas intestinalis are common parasites in the intestinal tract of healthy man in this part of Florida.

2. The finding of parasitic amebas and flagellates in the intestinal tract in cases of pellagra is purely coincidental. The parasites have nothing to do with the disease.

3. It is necessary to study stained specimens of every protozoon found in the intestine of man, before determining the species of the protozon and the question of its connection with disease.

4 . It is very probable that $E$. tetragena is the cause of at least a part of the amebic dysentery in the United States. ${ }^{1}$

1. Since writing the above, I have found $E$. tetragena in the stools of two colored persons who are suffering from dysentery and who have always lived here. I have also received a letter from Captain Craig, in which he informs me that he has found $E$. tetragena in material from Illinois; and $E$. tetragena has been reported, as a cause of dysentery in the United States, from the Mayos' laboratory at Rochester, Minn. 